\title{
Mirror Artifacts in Obstetric Ultrasound: Case Presentation of a Ghost Twin during the Second-Trimester Ultrasound Scan
}

\author{
Hyunyoung Ahn ${ }^{a, b}$ Edgar Hernández-Andrade ${ }^{a, b}$ Roberto Romero ${ }^{a, c, d}$ \\ Manasi Ptwardhan ${ }^{a, b}$ Luis F. Goncalves ${ }^{e}$ Alma Aurioles-Garibay ${ }^{a, b}$ \\ Maynor Garcia ${ }^{a, b}$ Sonia S. Hassan ${ }^{a, b}$ Lami Yeo $^{a, b}$
}

a Perinatology Research Branch, Program for Perinatal Research and Obstetrics, Division of Intramural Research, Eunice Kennedy Shriver National Institute of Child Health and Human Development, NIH, Bethesda, MD, and Detroit, MI, b Department of Obstetrics and Gynecology, Wayne State University School of Medicine, Detroit, Mich., 'Department of Obstetrics and Gynecology, University of Michigan, Ann Arbor, Ml, ${ }^{\mathrm{d}}$ Department of Epidemiology and Biostatistics, Michigan State University, East Lansing, MI, and ${ }^{\mathrm{e}}$ Departments of Obstetrics and Gynecology, and Radiology, Oakland University, William Beaumont School of Medicine, Rochester, Mich., USA

\section{Established Facts}

- Mirror artifacts are infrequent during obstetric ultrasound.

- The artificially duplicated image can increase the risk of misdiagnosis.

\section{Insights}

- A mirror artifact creating an extra fetus has not been previously reported at this advanced gestational age (18 weeks of gestation).

- This is the first report of a mirror artifact in obstetrics documented using both transabdominal and transvaginal ultrasound.

- This is the first report of synchronous but opposite movements between the real fetus and the mirrored image.

\section{Key Words}

Ultrasound physics · Twin gestation · Abdominal heterotopic pregnancy · Diagnostic error

\begin{abstract}
Mirror artifacts are produced by the reflection of ultrasound waves after they propagate through a structure and encounter a strong and smooth interface capable of acting as a mirror. Ultrasound waves bounce back and forth between the
\end{abstract}

\section{KARGER}

E-Mail karger@karger.com www.karger.com/fdt (c) 2013 S. Karger AG, Basel

$1015-3837 / 13 / 0344-0248 \$ 38.00 / 0$
Roberto Romero, MD, D. Med. Sci.

Perinatology Research Branch, NICHD, NIH, DHHS

Wayne State University/Hutzel Women's Hospital

3990 John R, Box 4, Detroit, MI 48201 (USA)

E-Mail romeror@mail.nih.gov 
mirroring interface and the reflective object and then eventually return to the transducer. The typical display of the mirror artifact consists of two similar structures separated and at similar distances from the reflective interface. We report a mirror artifact in a patient with a singleton gestation at 18 weeks. The image was interpreted as consistent with a twin gestation using transabdominal and transvaginal ultrasound. The differential diagnosis consisted of an abdominal heterotopic pregnancy. The presence of synchronized but opposite movements of both fetuses, and the blurred image of the second fetus, suggested a mirror artifact. The reflective surface was created by the interface located between a distended rectosigmoid filled with gas and the posterior uterine wall. Mirror artifacts can lead to diagnostic errors. This case illustrates how a distended rectosigmoid colon can generate an image that simulates either a twin gestation or an abdominal heterotopic pregnancy.

Copyright ๑ 2013 S. Karger AG, Basel

\section{Introduction}

Despite substantial advances in ultrasound imaging, diagnostic challenges continue to occur. An example of this is artifacts or errors in images that (1) cause structures to appear when they are not actually present or (2) result in a missing structure from the image or an improperly located representation of a structure with inadequate brightness, size, and shape [1-3].

Artifacts are inherent to ultrasound imaging and can occur regardless of the experience of the examiner and/or the proper use of technical settings. An example of such artifacts is called a mirror artifact; this is produced by reflection of the ultrasound waves after they propagate through a structure and encounter a strong, smooth interface acting as a mirror $[1,4]$. The waves bounce between the mirroring interface and the reflective object, and ultimately return to the transducer. These signals are displayed as a 'real' structure. However, because of the signal delay in arriving back to the transducer, the image representation on the ultrasound screen is deeper than the real structure and located at a similar distance from the reflective structure [1]. Typically, the mirror image is hypoechoic, blurred, and distorted compared to the image of the real structure, which has been attributed to the absorption and refraction of the reflected ultrasound signals. Mirror artifacts have been reported in vascular [46], abdominal [7], cardiac [8], and musculoskeletal [9, 10] ultrasound. Interestingly, a literature search showed that only 2 cases of mirror images have been reported in ob- stetrics $[11,12]$. Both were observed in early pregnancy, and the diagnostic challenge was related to an abdominal ectopic/heterotopic pregnancy. We report herein a case where a ghost fetus was observed due to a mirror artifact during a routine obstetrical ultrasound scan. The artifact was documented by transabdominal and transvaginal ultrasound.

\section{Case Presentation}

A 22-year-old woman, gravida 3 para 2, presented for an ultrasound scan at the Center for Advanced Obstetrical Care and Research (CAOCR - the Perinatology Research Branch of the Eunice Kennedy Shriver National Institute of Child Health and Human Development, National Institutes of Health, at Wayne State University School of Medicine and Hutzel Women's Hospital, Detroit, Mich., USA) at 18 weeks of gestation. The patient provided written informed consent for ultrasound examination and was enrolled in research protocols approved by the Institutional Review Boards of the National Institute of Child Health and Human Development, and by the Human Investigation Committee of Wayne State University.

The pregnancy was dated according to a reliable last menstrual period. The patient did not have a previous ultrasound examination. Her two prior pregnancies were uncomplicated and resulted in full-term vaginal deliveries. She had no history of medical/surgical complications. At the time of examination, the patient did not have any complaint - specifically, there was no abdominal pain, vaginal bleeding, or leaking of fluid. The patient reported a bloating sensation in her abdomen. Transabdominal ultrasound revealed an intrauterine pregnancy with fetal biometry consistent with her last menstrual period and normal fetal anatomy. During the course of the examination, another gestational sac was visible behind the first image. There appeared to be amniotic fluid and fetal movements within this second gestational sac, which was deeper than the first but adjacent to the posterior uterine wall. As the location of the second gestational sac appeared to be outside the uterine cavity, the diagnosis of an abdominal heterotopic abdominal pregnancy was considered (fig. 1; also see online suppl. videos 1 \& 2, www.karger.com/doi/10.1159/353702).

At the time of transvaginal ultrasound, a normally grown and active fetus was observed within the uterine cavity. The image suggesting the presence of an extrauterine gestational sac located behind the uterus in the rectouterine pouch containing fetal parts was noted. A complete anatomic survey of this extrauterine fetus was difficult, as clear images of the entire fetus could not be obtained. Two biometric parameters could be assessed: femur and humerus. Their length was similar to those of the fetus observed in the uterine cavity (fig. 2; also see online suppl. video 3). The lack of clinical symptoms and the inadequate visualization of the fetal anatomy prompted us to repeat the transabdominal ultrasound.

At this time, the image of the extrauterine fetus was obtained only when the patient was scanned at particular angles. Upon careful inspection, the movements of what was considered to be an extrauterine fetus were synchronous with those of the fetus within the uterus. Fetal movements of the 2 fetuses corresponded; name- 


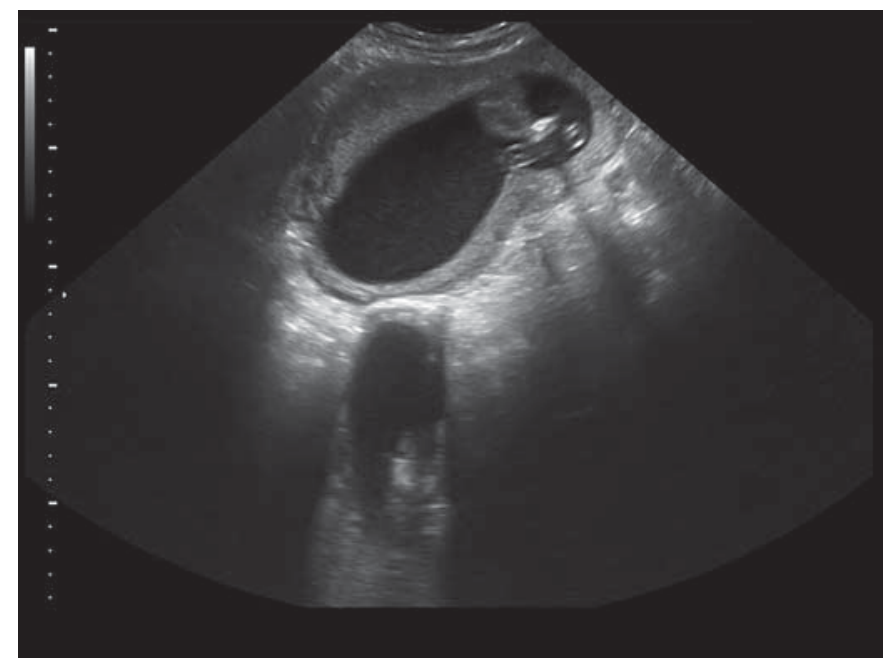

Fig. 1. Transabdominal ultrasound scan. The mirror artifact is observed behind the uterus. Note the high acoustic interphase between the bowel and the posterior wall of the uterus. The mirror artifact is only visible when the reflective surface is perpendicular to the ultrasound beam.

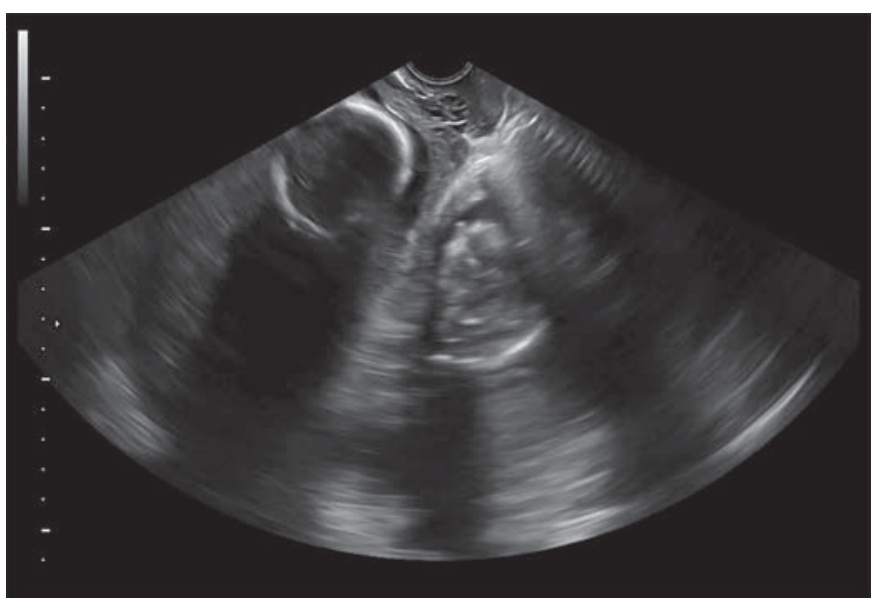

Fig. 2. Transvaginal ultrasound showing the reflection artifact creating the mirror image in the right side of the picture. The shape of the transvaginal ultrasound probe opens the emission of the ultrasound beam, the reflected signals travel at different angle as compared with the transabdominal ultrasound scan. The reflective surface is not completely perpendicular to the ultrasound beam, making the image more blurred and distorted.

ly, involving the same limb, but in the opposite direction. These movements were of similar amplitude but had a short time delay. Collectively, these findings raised the suspicion of a mirror artifact. When the ultrasound examination was repeated using different angles of insonation, the same findings were observed. A well-defined image of the second fetus could not be obtained in any of the scanned planes.
Since the patient had a bloated sensation, she was asked to evacuate her bowel. After this, we were no longer able to visualize the extrauterine fetus. A maternal MRI on the following day showed a single fetus within the uterus, and no evidence of an abdominal pregnancy.

\section{Discussion}

In the ultrasound artifact presented here, a maternal rectosigmoid colon containing a gas-fluid interface located immediately posterior to a thin uterine wall acted as a mirror. The reflection occurred between the normal intrauterine fetus and the gas-fluid interface, and eventually returned to the ultrasound transducer. The reflected echoes were delayed in their arrival due to the extra distance between the fetus and the uterine wall. This created a virtual fetus located behind the uterine wall (fig. 3). After bowel evacuation, the mirror image disappeared, as gas and fluid were no longer present to serve as a reflector.

Gas and fluid in the bowel were the main contributors to the ultrasound artifact, not only creating a strong interface with the posterior uterine wall, but also serving as a space of representation of the mirrored image in the ultrasound screen. Both signals, from the lumen of the bowel, and from the mirrored structure, reached the transducer at a similar time, creating the visual appearance of a fetus inside the bowel. As the reflected signals from the mirrored fetus were distorted, the image always appeared blurred. A second contributing factor was a thin posterior uterine wall. A posteriorly located placenta may increase the thickness of the posterior uterine wall, thus reducing the acoustic interface between the uterus and the bowel.

Good ultrasound mirrors are smooth surfaces with higher acoustic impedance, for example the pleura/lung interface, the hepatic-lung interface, air-containing abscesses, fluid/air interface in the stomach, and the urinary bladder $[3,7,13,14]$. In abdominal ultrasound, mirrored and blurred artifacts are frequently observed when the gastric tube is filled with gas [15]. In Doppler sonography, mirror artifacts have been reported in $2.5 \%$ of cases. This has been attributed to the fact that the wall and the lumen of the vessel can create a strong acoustic interphase [16, 17].

Despite the fact that mirror images can occur in different parts of the body, thus far only 2 cases have been reported in obstetrics. Lim et al. [11] reported a pregnant woman whose routine transabdominal ultrasound examination performed at 12 weeks was interpreted as diagnostic of a bilateral ectopic pregnancy ( 2 extrauterine fe- 


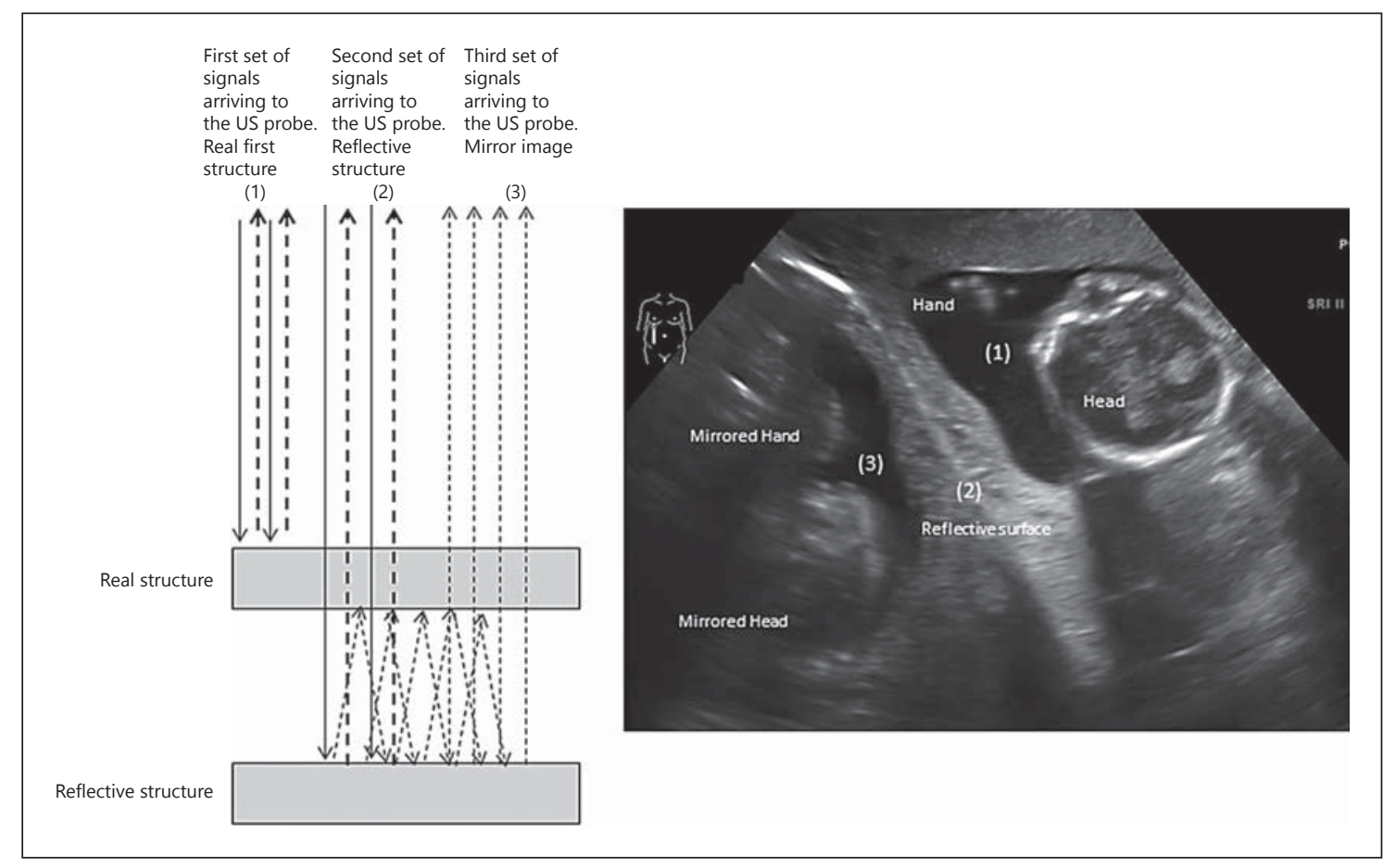

Fig. 3. Schematic representation of the mirror artifact. Ultrasound signals are normally reflected by the structure (fetal head), and by the reflective surface (posterior uterine wall and bowel wall) arriving on time to the transducer. Some ultrasound signals bounce back and forth between the head and the reflective surface finally arriving to the transducer. As they arrive later than the original signals, they are represented as another structure behind the reflective surface. tuses and 1 intrauterine fetus). A subsequent transvaginal ultrasound demonstrated that there was only a single intrauterine fetus with no evidence of the two extrauterine gestational sacs. In view of the ultrasound findings and the lack of clinical symptoms, the initial observations were considered artifacts. Miglietta et al. [12] described a case of a pregnant woman who underwent an ultrasound examination due to mild abdominal pain at 8 weeks of gestation. Transvaginal ultrasound showed an intrauterine sac with a normal fetus, and a second gestational sac with another active fetus was observed located in the rectouterine pouch. The authors made the diagnosis of an abdominal heterotopic pregnancy. Due to the lack of clinical symptoms, they performed a transabdominal ultrasound scan with a full bladder. The second gestational sac was no longer observed and the rectouterine pouch was visualized as free and normal.

Creation of an ultrasound image relies on some basic physical assumptions such as (1) the transmitted sound and the reflected echo travel in a straight line within the body, (2) the backscattered signals return after a single reflection, and (3) the time taken for the sound wave to travel to the reflector and back to the transducer determines the depth of the structure $[3,18,19]$. Only a small fraction of the ultrasound waves are reflected and the majority are either refracted or transmitted [20]. When these assumptions are not met, multiple echo pathways, and velocity and attenuation errors can generate imaging artifacts such as reverberation (several lines and an equidistant space along a ray line), ring-down artifacts (solid structures that vibrate and enhance the signals traveling deeper in the structure), enhancement, attenuation and the mirror, and reflected artifacts described herein $[2,3,19,21]$.

If an ultrasound artifact is recognized, the examiner should try to correct it by adjusting the ultrasound probe frequency, energy output, and gain settings, using tissue harmonics and/or changing the orientation of the transducer $[5,16]$. If ultrasound artifacts are not promptly recognized, they may lead to diagnostic errors such as the presence of abdominal ectopic or heterotopic pregnancies in obstetric ultrasound $[11,12]$, images suggesting an ulcerated embolus created by a stenotic vessel during vascular Doppler sonography [22], a false reinforcement behind hepatic angiomas during abdominal ultrasound [23], an artificially created tracheal mass due to a thyroid nodule during an ultrasound scan of the neck [24], and 
images suggesting malignant infiltration in patients with rectal tumors during pelvic ultrasound [25].

In our case, the ultrasound image raised the suspicion of a second fetus located immediately posterior to the uterus, although it was never possible to obtain a clear image of such a fetus. Also, the clinical symptoms of the patient did not match the ultrasound findings. The presence of synchronic and opposite fetal movements oriented to the identification of a mirror artifact. MRI performed one day later excluded any possibility of an abdominal heterotopic pregnancy. The patient had an uncomplicated pregnancy and delivered a normal, full-term, and healthy neonate.

\section{Conclusion}

During obstetric ultrasound, image artifacts can be produced. A proper recognition of these artifacts can reduce the risk of misdiagnosis.

\section{Acknowledgements}

This project has been funded in whole or in part with Federal funds from the Eunice Kennedy Shriver National Institute of Child Health and Human Development, National Institutes of Health, Department of Health and Human Services, under Contract No. HHSN275201300006C.

\section{References}

1 Feldman MK, Katyal S, Blackwood MS: US ar- 10 Antonakakis JG, Sites B: The five most comtifacts. Radiographics 2009;29:1179-1189.

$\checkmark 2$ Keogh CF, Cooperberg PL: Is it real or is it an artifact. Ultrasound Q 2001;17:201-210.

3 Kremkau F: Artifacts; in Kremkau F (ed): Diagnostic Ultrasound Principles and Instruments, ed 7. New York, Elsevier, 2006, pp 261-298.

$\checkmark 4$ Hebbard PD: Artifactual mirrored subclavian artery on ultrasound imaging for supraclavicular block. Can J Anaesth 2009;56:537538.

5 Rubin JM, Gao J, Hetel K, Min R: Duplication images in vascular sonography. J Ultrasound Med 2010;29:1385-1390.

$\checkmark 6$ Middleton WD, Melson GL: The carotid ghost. A color Doppler ultrasound duplication artifact. J Ultrasound Med 1990;9:487-493.

7 Grech P: Mirror-image artifact with endoscopic ultrasonography and reappraisal of the fluid-air interface. Gastrointest Endosc 1993; 39:700-703.

-8 Linka AZ, Barton M, Attenhofer Jost C, Jenni $\mathrm{R}$ : Doppler mirror image artifacts mimicking mitral regurgitation in patients with mechanical bileaflet mitral valve prostheses. Eur J Echocardiogr 2000;1:138-143.

$>9$ McDonald S, Fredericson M, Roh EY, Smuck $\mathrm{M}$ : Basic appearance of ultrasound structures and pitfalls. Phys Med Rehabil Clin N Am 2010;21:461-479. mon ultrasound artifacts encountered during ultrasound-guided regional anesthesia. Int Anesthesiol Clin 2011;49:52-66.

11 Lim BH, Amos M, Fairhead AC: The mirror image artifact of early pregnancy. Ultrasound Obstet Gynecol 2003;21:518-520.

12 Miglietta F, D’Antonio F, Matarrelli B, Ianieri MM, Liberati M, Celentano C: Mirror-image artifact of early pregnancy on transvaginal sonography. J Ultrasound Med 2012;31:18581859.

13 Mihmanli I, Cetinkaya S, Kurugoglu S, Kantarci F, Esen G: Another face of mirror-image artifact. Eur J Ultrasound 2001;14:183-185.

14 Sandler MA, Madrazo BL, Walter R, Beute GH, Hudak SF, Haggar AH, Maywood CM: Ultrasound case of the day. Duplication artifact (mirror image artifact). Radiographics 1987;7:1025-1028.

15 Wilson SR, Burns PN, Wilkinson LM, Simpson DH, Muradali D: Gas at abdominal US appearance, relevance, and analysis of artifacts. Radiology 1999;210:113-123.

16 Arning C, Eckert B: The diagnostic relevance of colour Doppler artefacts in carotid artery examinations. Eur J Radiol 2004;51:246-251.
17 Gustavson S, Olin JW: Images in vascular medicine. Mirror image artifact. Vasc Med 2006;11:175-176.

18 Kossoff G: Basic physics and imaging characteristics of ultrasound. World J Surg 2000;24: 134-142.

19 Aldrich JE: Basic physics of ultrasound imaging. Crit Care Med 2007;35:S131-S137.

20 Lieu D: Ultrasound physics and instrumentation for pathologists. Arch Pathol Lab Med 2010;134:1541-1556.

21 Scanlan KA: Sonographic artifacts and their origins. Am J Roentgenol 1991;156:1267-1272.

22 Arning C: Mirror image artifacts of color Doppler images causing misinterpretation in carotid artery stenoses. J Ultrasound Med 1998;17:683-686.

23 Peetrons P, Delplace O, Ermens N: Posterior reinforcement behind hepatic angiomas. Mirror artefact as a possible source of error. J Radiol 1988;69:117-122.

24 Diazzi C, Gnarini V, Brigante G, Rochira V: Ultrasound mirror artifact of a thyroid nodule by trachea mimicking a tracheal mass. Thyroid 2011;21:929-930.

25 Hulsmans FJ, Castelijns JA, Reeders JW, Tytgat GN: Review of artifacts associated with transrectal ultrasound: understanding, recognition, and prevention of misinterpretation. J Clin Ultrasound 1995;23:483-494. 